\title{
Evaluación de las concentraciones de Physalis peruviana y Stevia rebaudiana liofilizada en una bebida instantánea
}

\section{Evaluation of the concentrations of Physalis peruviana and lyophilized Stevia rebaudiana in an instant drink}

\author{
Jessica Chuquizuta Reyna ${ }^{1}$, Erick Aldo Auquiñivin Silva ${ }^{2}$
}

\section{RESUMEN}

El objetivo del estudio fue evaluar las concentraciones de Physalis peruviana "aguaymanto" y Stevia rebaudiana "stevia" liofilizada en la aceptabilidad de una bebida instantánea, para lo cual se aplicó 5\%, 8\% y 10\% de aguaymanto liofilizado y $0.08 \%, 0.10 \%$ y $0.12 \%$ de stevia liofilizada. Se utilizó un diseño experimental DCA con 9 tratamientos. El análisis sensorial se realizó mediante una escala hedónica de 5 puntos, con un panel semi entrenado de 12 personas que evaluaron los atributos de aroma, sabor y color. Las características fisicoquímicas evaluadas en las bebidas fueron: $\%$ acidez total, $\mathrm{pH}$ y ${ }^{\circ}$ Brix, encontrándose en ellas diferencia significativa $(\mathrm{P}<0.05)$ en los 9 tratamientos. Según la separación de medias Tukey no se mostraron diferencias significativas $(\mathrm{P}<0.05)$ en la evaluación sensorial en los atributos de aroma y sabor, pero si se observaron diferencias significativas $(\mathrm{P}<0.05)$ en el color de la bebida. El tratamiento que tuvo mayor calificación con respecto al análisis sensorial fue el T6, en la cual la concentración de aguaymanto y stevia fue de $8 \%$ y $0.12 \%$ respectivamente, en una bebida de $250 \mathrm{ml}$. La calificación de este tratamiento según la escala hedónica fue de "me gusta poco", con valor de 3.95 ; con una acidez total de $1.02 \%, \mathrm{pH}: 3.50 \mathrm{y}^{\circ}$ Brix: 7.88 .

Palabras clave: Aguaymanto, liofilización, stevia, bebida instantánea.

\begin{abstract}
The objective of the study was to evaluate the concentrations of Physalis peruviana "aguaymanto" and lyophilized Stevia rebaudiana "stevia" in the acceptability of an instant drink, for which 5\%, 8\% and 10\% of lyophilized aguaymanto and $0.08 \%, 0.10$ were applied $\%$ and $0.12 \%$ lyophilized stevia. A DCA experimental design with 9 treatments was used. Sensory analysis was performed using a 5-point hedonic scale, with a semi-trained panel of 12 people who evaluated the attributes of aroma, flavor and color. The physicochemical characteristics evaluated in the drinks were:\% total acidity, $\mathrm{pH}$ and ${ }^{\circ}$ Brix, finding a significant difference $(\mathrm{P}<0.05)$ in the 9 treatments. According to the separation of Tukey means, no significant differences $(\mathrm{P}<0.05)$ were shown in the sensory evaluation in the aroma and flavor attributes, but significant differences $(\mathrm{P}<0.05)$ were observed in the color of the drink. The treatment that had the highest rating with respect to the sensory analysis was T6, in which the concentration of aguaymanto and stevia was $8 \%$ and $0.12 \%$ respectively, in a $250 \mathrm{ml}$ drink. The qualification of this treatment according to the hedonic scale was "I like it little", with a value of 3.95; with a total acidity of $1.02 \%, \mathrm{pH}$ : 3.50 and $^{\circ}$ Brix: 7.88 .
\end{abstract}

Keywords: Aguaymanto, lyophilization, stevia, instant drink.

Bachiller Ingeniería Agroindustrial. Universidad Nacional Toribio Rodríguez de Mendoza de Amazonas. Correo electrónico: jesyreyna05@gmail.com

${ }^{2}$ MSc. Ingeniería Agroindustrial. Universidad Nacional Toribio Rodríguez de Mendoza de Amazonas. Correo electrónico:erick.auquinivin@untrm.edu.pe 


\section{INTRODUCCIÓN}

En la región Amazonas existen muchas frutas nativas entre ellas: el aguaymanto, purpur, papayita de monte, pepino de árbol y el babaco, con alto contenido de nutrientes y propiedades organolépticas, con la técnica de liofilizado se logró obtener materia prima seca; como ingredientes en la industrialización de bebidas saludables, compotas, salsas, papillas, gelatinas con pulpa, buscando permanecer su composición nutricional, su altísima hidratación y la tipicidad de sus sabores o aromas (Santillán, 2013).

Según el INEI-Encuesta Nacional de presupuesto Familiar 2008-2009, en la ciudad de Chachapoyas el consumo promedio per cápita anual de bebidas gasificadas es 16,5 litros; este dato se debe porque son bebidas que ya tienen un mercado ganado, hábitos de consumo y porque no existen otras bebidas competitivas. Con este trabajo se obtuvo una bebida alternativa que mostró la calidad y garantía al ser consumida.

La liofilización es un proceso de conservación mediante sublimación utilizado con el fin de reducir las pérdidas de los componentes volátiles o termosensibles, es el más noble proceso de conservación de productos biológicos conocidos, sin conservantes o productos químicos, es el proceso más adecuado para preservar células, enzimas, vacunas, virus, levaduras, sueros, derivados sanguíneos, algas, así como frutas, vegetales, carnes, peces y alimentos en general. En este proceso de secado los productos obtenidos no se ven alterados en sus propiedades y se rehidratan fácilmente (Alvarado, 1996).

Las bebidas lácteas instantáneas son mezclas deshidratadas, en polvo o granuladas, elaboradas con una relación mínima de $30 \%$ de leche en el producto final, puede contener agregados de otros ingredientes de uso alimentarios permitidos, entre ellos, cereales, azúcares, saborizantes de frutas, chocolate, vainilla, edulcorantes, frutas o vegetales deshidratadas (Covenin,2001).

La porción de bebida nutritiva instantánea es de $32 \mathrm{~g}$ diluida en $200 \mathrm{ml}$ de agua, recomendando agregar 23 $\mathrm{g}$ de azúcar para que la bebida tenga $12{ }^{\circ}$ Brix. Inestroza (2003).

Según Vargas (2015), la cocona es una fruta de la Amazonía que al ser liofilizada se retiene su valor nutritivo, disminuye costos de almacenamiento y aumenta tiempo de vida útil del producto, lo cual puede garantizar que la cocona en polvo sea un producto de exportación. Realizó una investigación para evaluar el efecto del proceso de liofilización sobre propiedades fisicoquímicas y vida útil de cocona (Solanum sessiliflorum Dunal) en polvo.
Se recomienda consumir una dosis diaria de $8 \mathrm{mg}$ stevia/ $\mathrm{kg}$ de peso, para una persona con un peso de 75 $\mathrm{kg}$ la dosis diaria no debe de sobrepasar los $600 \mathrm{mg}$ al día, mientras que para una persona con un peso de 69 $\mathrm{kg}$, la dosis máxima es de $552 \mathrm{mg}$ al día.

Según EFSA Journal (2010), establece que la ingesta diaria admisible para los glicósidos de esteviol, que se extraen de la hoja de la stevia, se expresa como equivalentes del esteviol siendo de $4 \mathrm{mg} / \mathrm{kg}$ de peso corporal al día. Esto equivale aproximadamente a 12 $\mathrm{mg}$ de extractos de stevia de alta pureza/ $\mathrm{kg}$ de peso corporal al día.

\section{MATERIAL Y MÉTODO}

\subsection{Desarrollo de la de bebida instantanea}

A partir de jugo liofilizado de aguaymanto se elaboró la bebida, utilizando una proporción agua, aguaymanto liofilizado (AL) y stevia liofilizado (SL). Donde se utilizó un diseño factorial 3k (superficie de respuestas), donde $\mathrm{K}$ es el número de variables en estudio (AL y SL):

Aguaymanto liofilizado: 5\%, 8\% y 10\% en relación al peso de la parte liquida (agua).

Stevia: $0.08 \%, 0.10 \%$ y $0.12 \%$ en relación al peso de la parte liquida (agua).

Asimismo, se obtuvo como variables de respuesta: $\mathrm{pH}, \%$ de acidez total $\mathrm{y}{ }^{\circ}$ Brix.

\subsection{Formulación de la bebida instantánea}

a. Recolección: Se recolectaron del mercado central de Chachapoyas tomando en cuenta la madurez fisiológica y aceptada para el consumo.

b. Limpieza: Esta operación se efectuó en forma manual eliminando los restos de tierra y/o partículas extrañas que puedan estar adheridas a su superficie.

c. Lavado: Luego de la operación de limpieza se procedió a un lavado inmediato con abundante agua, con la finalidad de evitar la formación de costras en superficie a causa de la soda empleada, las cuales una vez que se forman son difíciles de removerlas.

d.Acabado: En esta operación se procedió a efectuar el recorte de la forma transversal para ayudar al licuado.

e. Licuado: Consistió en la inmersión de los frutos de aguaymanto en la licuadora.

f. Congelación: Aquí el jugo de aguaymanto pasó a un sistema de congelación de $4^{\circ} \mathrm{Cx} 24$ horas.

g. Liofilización: Una vez congelada el aguaymanto se inició el proceso de liofilización.

h. Mezcla: Se mezcló el aguaymanto liofilizado con el edulcorante liofilizado de Stevia de acuerdo al tratamiento definido.

i. Envasado: Las muestras liofilizadas se colocó en envases de aluminada de polietileno.

j. Almacén: Se conservó a temperatura ambiente, seca y ventilada.

k. Elaboración de la Bebida instantánea: En 250 
ml de agua se colocó la concentración de aguaymanto y stevia liofilizado de acuerdo a los tratamientos definidos.

\subsection{Análisis Fisicoquímicos}

Se realizó tres veces la determinación de cada análisis fisicoquímico:

a. Análisis de Humedad siguiendo la metodología sugerida por la AOAC $925.45 \mathrm{~B}$.

b. Solidos Solubles Totales ( ${ }^{\circ}$ Brix). Refractómetro (0 a $32^{\circ}{ }^{\circ}$ Brix).

c. Acidez total. Método Oficial 942.15. AOAC.

d.pH. Las medidas de $\mathrm{pH}$ se realizan en el zumo a temperatura ambiente, empleando un $\mathrm{pH}$-metro digital calibrado con soluciones tampón de $\mathrm{pH}=4.00$ y $\mathrm{pH}=7.02$.

\subsection{Evaluación Sensorial}

Se usaron 12 panelistas (semi capacitados) en la evaluación sensorial. Se efectuaron 12 repeticiones del panel para cada tratamiento en un solo día. Se repartió $60 \mathrm{ml}$ por tratamiento a cada panelista; se controló la temperatura del laboratorio a $20^{\circ} \mathrm{C}$ y se repartieron galletas soda y agua para neutralizar el paladar. Las características sensoriales evaluadas fueron las siguientes: Aroma, apariencia, acidez, viscosidad, sabor y aceptación general. Se evaluaron los 9 tratamientos con una escala hedónica de 5 puntos:

1. Me disgusta mucho

2. Me disgusta poco

3. Ni me gusta ni me disgusta

4. Me gusta un poco

5. Me gusta mucho

\subsection{Diseño Experimental.}

Se utilizó un diseño Completos al Azar (DCA), con 9 tratamientos y una repetición, para un total de 12 panelistas.

Se efectuó un análisis de varianza (ANDEVA) para analizar la información obtenida en los análisis sensoriales. Una separación de medias Tukey $(\mathrm{P}<$ 0.05) fue utilizada para determinar diferencias estadísticas entre los tratamientos. Se usó el Sistema de Análisis Estadístico (INFOSTAT), Versión 2017.

A continuación, se detalla la concentración de pulpa de Aguaymanto liofilizado son 5\%, 8\% y $10 \%$ y Stevia liofilizada $0.08 \%, 0.10 \%$ y $0.12 \%$.

\section{Análisis comparativo}

\begin{tabular}{|c|c|c|c|c|c|c|c|c|c|}
\hline A & \multicolumn{3}{|c|}{ A1 } & \multicolumn{3}{c|}{ A2 } & \multicolumn{3}{c|}{ A3 } \\
\hline B & B1 & B2 & B3 & B1 & B2 & B3 & B1 & B2 & B2 \\
\hline 1 & A1B1 & A1B2 & A1B3 & A2B1 & A2B2 & A2B3 & A3B1 & A3B2 & A3B3 \\
\hline & T1 & T2 & T3 & T4 & T5 & T6 & T7 & T8 & T9 \\
\hline
\end{tabular}

Leyenda:

A(\% de liofilización de Aguaymanto).
B (\% de Stevia liofilizada).

\section{RESULTADOS}

Tabla 1. Análisis biométrico del aguaymanto

\begin{tabular}{|c|c|c|}
\hline \multirow{2}{*}{ AGUAYMANTO } & PESO & DIAMETRO \\
\cline { 2 - 3 } & $\mathbf{4 . 7 6}$ & $\mathbf{1 9 .}$ \\
\hline
\end{tabular}

Tabla 2. Composición fisicoquímica del aguaymanto fresco

\begin{tabular}{|l|c|c|c|l|}
\hline Densidad & $\mathbf{p H}$ & \%Ac & ${ }^{\circ}$ Brix & \%H \\
\hline 1.087 & 3.9 & $\mathbf{1 . 6}$ & 13.4 & 73.74 \\
\hline
\end{tabular}

Tabla 3. Composición fisicoquímica del aguaymanto liofilizado

\begin{tabular}{c|c|c|r|r|}
\hline pH & $\%$ Ac & ${ }^{\circ}$ Brix & $\%$ \% & \\
\hline 3.5 & 2.3 & 15.0 & 20.00 & \\
\hline
\end{tabular}

Tabla 4. Rendimiento del aguaymanto

Ingrediente

Cantidad (100\%)

\begin{tabular}{ll}
\hline Agua & Enrasado hasta $250 \mathrm{ml}$. \\
Aguaymanto Liof. & $* 5,8$ y $10 \%$ \\
Stevia & $* 0.08,0.10$ y $0.12 \%$ \\
\hline
\end{tabular}

Tabla 5. Formulación de la bebida de aguaymanto con stevia

\begin{tabular}{lc}
\hline \multicolumn{1}{c}{ Aguay manto (100\%) } \\
\hline $\begin{array}{l}\text { Pulpa en base a la materia } \\
\text { Prima. }\end{array}$ \\
$\begin{array}{l}\text { Liofilizado en base a la } \\
\text { pulpa }\end{array}$ & $10.05 \%$ \\
\hline
\end{tabular}

* Porcentaje en relación al total de la bebida.

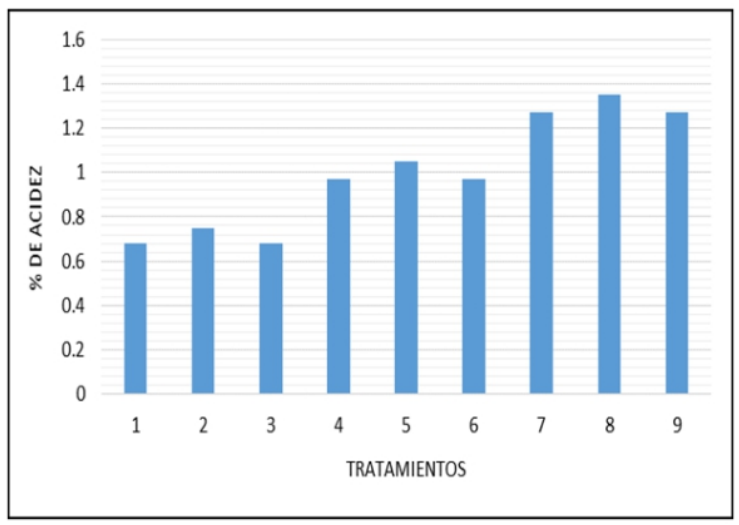

Figura 1. Comportamiento de la acidez, respeto a los tratamientos. 


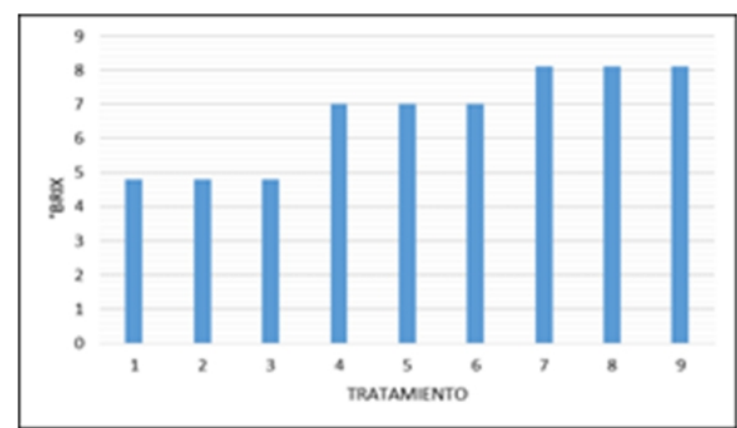

Figura 2. Comportamiento del ${ }^{\circ}$ Brix, respecto a los tratamientos.

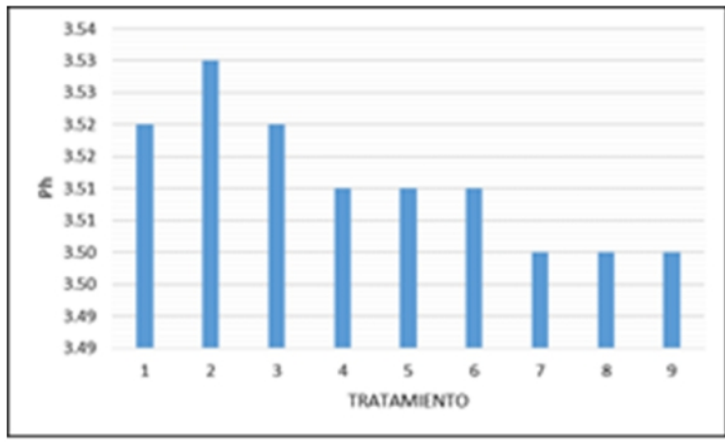

Figura 3. Comportamiento del $\mathrm{pH}$, respecto a los tratamiento sensoriales de aroma, sabor y color.

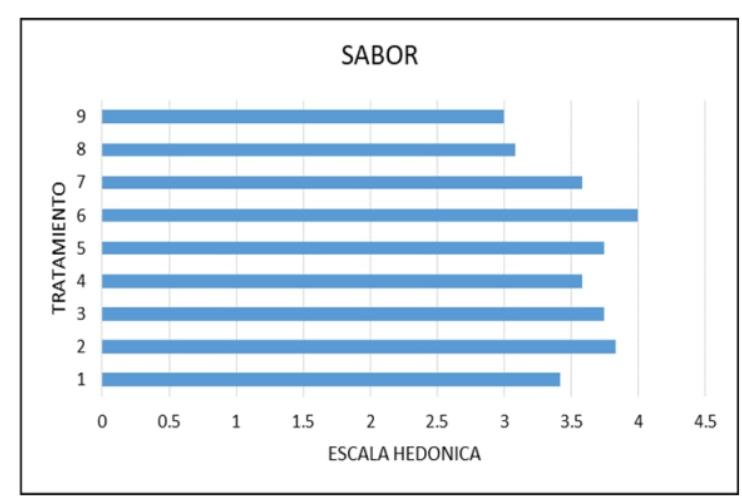

Figura 4. Comportamiento del atributo sabor, respecto a los tratamientos.

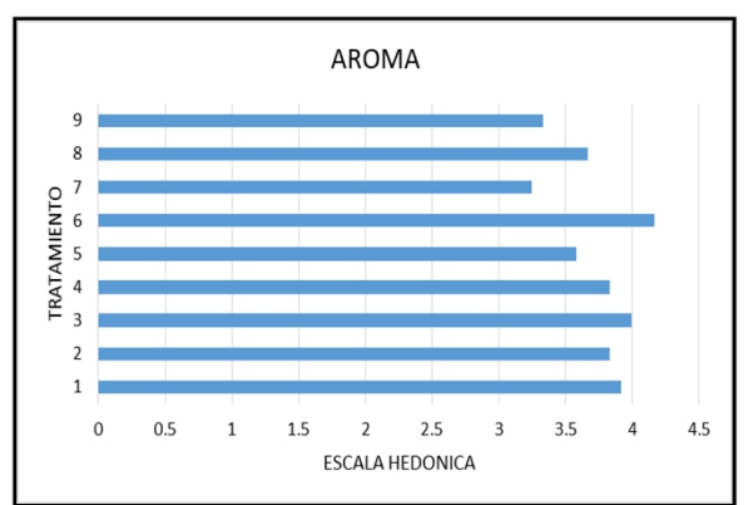

Figura 5. Comportamiento del atributo aroma, respecto a los tratamientos

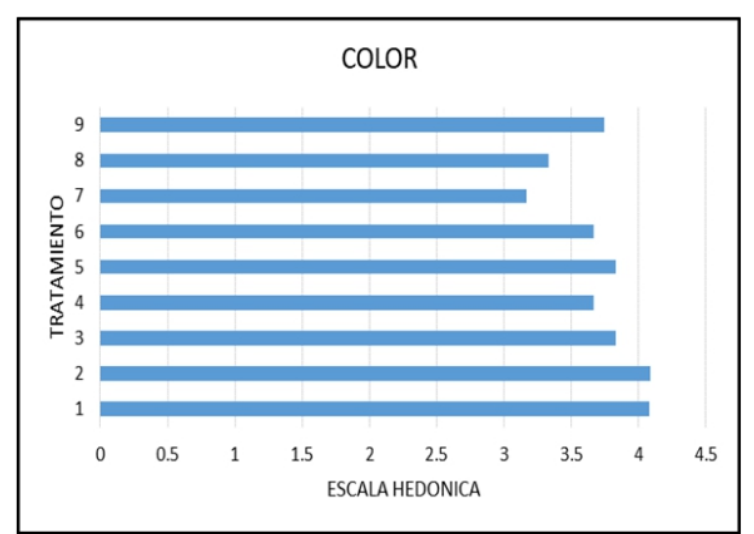

Figura 6. Comportamiento del atributo Color, respecto a los tratamientos.

Tabla 6. Calificación de general del análisis sensorial y fisicoquímico en los tratamientos.

\begin{tabular}{|c|c|c|c|c|c|c|c|c|}
\hline \multirow{2}{*}{ 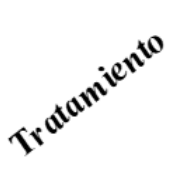 } & \multirow{2}{*}{$\begin{array}{c}\% \text { de } \\
\text { Aguaymanto } \\
\text { liofilizado y } \\
\text { Stevia }\end{array}$} & \multicolumn{4}{|c|}{ Califacción según escala Hedónica } & \multirow{2}{*}{$\% A c$} & \multirow{2}{*}{$p H$} & \multirow{2}{*}{${ }^{\circ}$ Brix } \\
\hline & & Color & Sabor & Aroma & Promedio & & & \\
\hline 1 & $5 ; 0.08$ & 4.08 & 3.42 & 3.92 & 3.81 & 0.71 & 3.54 & 4.8 \\
\hline 2 & $5 ; 0.10$ & 4.09 & 3.83 & 3.83 & 3.92 & 0.74 & 3.51 & 4.8 \\
\hline 3 & $5 ; 0.12$ & 3.83 & 3.75 & 4 & 3.86 & 0.81 & 3.5 & 4.8 \\
\hline 4 & $8 ; 0.08$ & 3.67 & 3.58 & 3.83 & 3.69 & 0.92 & 3.49 & 7.88 \\
\hline 5 & $8 ; 0.10$ & 3.83 & 3.75 & 3.58 & 3.72 & 0.97 & 3.49 & 7.89 \\
\hline 6 & $8 ; 0.12$ & 3.67 & 4 & 4.17 & 3.95 & 1.02 & 3.5 & 7.88 \\
\hline 7 & $10 ; 0.08$ & 3.17 & 3.58 & 3.25 & 3.33 & 1.16 & 3.5 & 10.1 \\
\hline 8 & $10 ; 0.10$ & 3.33 & 3.08 & 3.67 & 3.36 & 1.26 & 3.5 & 10.11 \\
\hline 9 & $10 ; 0.12$ & 3.75 & 3 & 3.33 & 3.36 & 1.3 & 3.5 & 10.1 \\
\hline
\end{tabular}


En esta tabla podemos resaltar que el tratamiento 6 tiene la calificación de 3.95 respecto a la escala hedónica que corresponde a la calificación de "me gusta un poco".

\section{DISCUSIÓN}

Al convertir los datos de muestra fresca y comparar con las liofilizadas, se aprecia diferencia significativa en la determinación de acidez y pH. Se debe tener en cuenta que las frutas contienen ácidos orgánicos de forma libre o combinada que están disueltos en las vacuolas de las células que al eliminar el agua se concentrarían. (Huaraca 2011).

La bebida instantánea aceptada tiene una porción de $20.3 \mathrm{~g}$ diluido en $250 \mathrm{ml}$ de agua, semejante al propuesto por Inestroza (2003), donde él elabora una bebida nutritiva instantánea con 32 g diluida en 200 $\mathrm{ml}$ de agua. Así mismo recomienda agregar $23 \mathrm{~g}$ de azúcar para que la bebida tenga $12^{\circ}$ Brix. Por lo cual en nuestro caso solo se agregó $20 \mathrm{~g}$ de Aguaymanto liofilizada y $0.3 \mathrm{~g}$ de Stevia liofilizada, donde se obtuvo un $7.88^{\circ}$ Brix; $1.02 \%$ Acidez total y $3.5 \mathrm{pH}$.

Los grados ${ }^{\circ}$ Brix y $\mathrm{pH}$, en bebidas instantáneas, según NTP está entre $8-15^{\circ}$ Brix y en pH está entre 3.5 a 7, respectivamente. Los resultados obtenidos del ${ }^{\circ}$ Brix y $\mathrm{pH}$, están dentro de los rangos establecidos por la Normas Técnicas Peruanas, 209.038:2009.

\section{CONCLUSIONES}

Se desarrollaron las formulaciones y el diagrama de flujo de proceso a nivel piloto de la bebida de liofilizada de Aguaymanto con niveles de: $5 \%, 8 \%$ y $10 \%$ y Stevia liofilizada con niveles de: $0.08 \%$, $0.10 \%$ y $0.12 \%$

No se encontraron diferencias estadísticamente significativas en la evaluación sensorial respecto al Aroma y Sabor, pero si existieron diferencias estadísticamente significativas en el color los panelistas indicaron que le tratamiento 6 posee mejor aceptación ya que la puntuación fue mayor en las tres características sensoriales, esto indica que se podría elaborar a mayor escala según la aceptación descrita en el trabajo de investigación.

Los niveles de aguaymanto y stevia liofilizada en la bebida, generaron diferencia significativa en los valores de $\%$ de acidez total, grados ${ }^{\circ} \mathrm{Brix}$ y $\mathrm{pH}$.

El proceso de liofilización en el aguaymanto afectó significativamente al $\%$ de acidez total, observándose un incremento con respecto a la muestra fresca, en $143.75 \%$, con una humedad de $20 \%$.

Se determinó que el tratamiento T6 fue el que presentó mayor calificación según la escala hedónica, con una aproximación de 4, que se interpreta como "Me gusta poco".

\section{REFERENCIA BIBLIOGRÁFICA}

Alvarado, J. de D. (1996). Principios de Ingeniería Aplicada a Alimentos. Ed. Radio Comunicaciones OEA, Quito, Ecuador.

Covenin 2001. (Comisión Venezolana de Normas Industriales. Mezclas deshidratadas para preparar bebidas instantáneas. $\mathrm{n}^{\circ}$ 2125:2001. 1era. Revisión. Publicación de Fondo Norma, Categoría B. ICS. 67.260.20. Caracas, Venezuela.p 13, 2001.

EFSA Journal, 2010. Global Stevia Institute. D i s p n i b l e e n : http://globalsteviainstitute.com/es/laciencia-de-la-stevia/seguridad-de-la-stevia/

INEI, 2008-2009. Encuesta Nacional de presupuesto Familiar.

Inestroza, BS. 2003. Desarrollo y evaluación de una bebida nutricional instantánea para niños en edad escolar. Tesis Lic. Ing. Aig. Honduras. Zamorano. $40 \mathrm{p}$.

Huaraca A. 2011. Evaluación nutritiva y nutracéutica de la frutilla (Fragaria vesca) deshidratada por el método de liofilización y comparación con la obtenida por deshidratación en microondas. RiobambaEcuador: Escuela Superior Politécnica de Chimborazo. Facultad de Ciencias - Escuela de Bioquímica y Farmacia.

NTC. 2010.Mezclas en polvo para preparar refrescos o bebidas instantáneas. requisitos. $\mathrm{N}^{\circ} 5767$.

Santillán, F. 2013. Plan de negocio producción y comercialización de papa (semilla y consumo) de la zona de luya en la región nororiental del Perú. Chachapoyas.

Vargas, 2015. Efecto de la liofilización sobre propiedades fisicoquímicas y vida útil de cocona (Solanum sessiliflorum Dunal) en polvo. Universidad Nacional de Colombia Sede Palmira. 